\title{
Neurological emergencies associated with COVID-19: stroke and beyond
}

\author{
Amit Agarwal ${ }^{1}$ D $\cdot$ Marco Pinho $^{1} \cdot$ Karuna Raj $^{1} \cdot$ Frank F. Yu $^{1} \cdot$ Girish Bathla $^{2} \cdot$ Michael Achilleos $^{1} \cdot$ Thomas ONeill $^{1}$. \\ Michael Still ${ }^{3} \cdot$ Joseph Maldjian ${ }^{1}$
}

Received: 8 July 2020 / Accepted: 3 August 2020 / Published online: 11 August 2020

(C) American Society of Emergency Radiology 2020

\begin{abstract}
Novel coronavirus disease (COVID-19) was declared a global pandemic on March 1, 2020. Neurological manifestations are now being reported worldwide, including emergent presentation with acute neurological changes as well as a comorbidity in hospitalized patients. There is limited knowledge on the neurologic manifestations of COVID-19 at present, with a wide array of neurological complications reported, ranging from ischemic stroke to acute demyelination and encephalitis. We report five cases of COVID-19 presenting to the ER with acute neurological symptoms, over the course of 1 month. This includes two cases of ischemic stroke, one with large-vessel occlusion and one with embolic infarcts. The remainders of the cases include acute tumefactive demyelination, isolated cytotoxic edema of the corpus callosum with subarachnoid hemorrhage, and posterior reversible encephalopathy syndrome (PRES).
\end{abstract}

Keywords COVID-19 $\cdot$ Neurological $\cdot$ MRI $\cdot$ Hypercoagulable $\cdot$ Neurotropism

\begin{tabular}{|c|c|c|c|}
\hline Abbreviations & & \multicolumn{2}{|r|}{ von Willebrand factor } \\
\hline \multirow[t]{2}{*}{ SARS-CoV-2 } & Severe Acute Respiratory & \multicolumn{2}{|r|}{ Angiotensin-converting enzyme 2} \\
\hline & Syndrome-coronavirus 2 & & \\
\hline $\begin{array}{l}\text { COVID-19 } \\
\text { RT-PCR }\end{array}$ & Coronavirus disease 2019 & & \\
\hline RT-PCR & $\begin{array}{l}\text { Real-time reverse } \\
\text { transcriptase-polymerase chain reaction }\end{array}$ & \multicolumn{2}{|c|}{ Introduction } \\
\hline \multirow{5}{*}{$\begin{array}{l}\text { CSF } \\
\text { IL1, IL-6 } \\
\text { TNF } \alpha \\
\text { tPA } \\
\text { ID }\end{array}$} & Cerebrospinal fluid & \multirow{5}{*}{\multicolumn{2}{|c|}{$\begin{array}{l}\text { The novel SARS-CoV-2 was first identified on January } 7 \text {, } \\
2020 \text {, in a patient with atypical pneumonia and was declared } \\
\text { a global pandemic within } 2 \text { months of the first identified case. } \\
\text { Similar to other coronaviruses, SARS-CoV-2 primarily affects } \\
\text { the respiratory system and presents with respiratory distress. }\end{array}$}} \\
\hline & Interleukin 1,6 & & \\
\hline & Tumor necrosis factor-alpha & & \\
\hline & Tissue plasminogen activator & & \\
\hline & Infectious disease & & \\
\hline \multirow{2}{*}{\multicolumn{2}{|c|}{$\begin{array}{l}\triangle \quad \text { Amit Agarwal } \\
\text { amit.agarwal@utsouthwestern.edu }\end{array}$}} & Thor & \\
\hline & & Thol & 1@utsouthwestern.edu \\
\hline \multirow{2}{*}{\multicolumn{2}{|c|}{$\begin{array}{l}\text { Marco Pinho } \\
\text { Marco.pinho@utsouthwestern.edu }\end{array}$}} & Micl & \\
\hline & & Micl & @utsouthwestern.edu \\
\hline \multirow{2}{*}{\multicolumn{2}{|c|}{$\begin{array}{l}\text { Karuna Raj } \\
\text { Karuna.raj@utsouthwestern.edu }\end{array}$}} & Jose & \\
\hline & tsouthwestern.edu & Jose & ian@utsouthwestern.edu \\
\hline \multicolumn{2}{|c|}{ Frank F. Yu } & & \\
\hline \multicolumn{2}{|c|}{ Frankf.yu@utsouthwestern.edu } & $\begin{array}{l}\text { Univ } \\
\text { Blvd }\end{array}$ & $\begin{array}{l}\text { Texas Southwestern Medical Center, } 5323 \text { Harry Hines } \\
\text { TX } 75390 \text {, USA }\end{array}$ \\
\hline \multicolumn{2}{|c|}{$\begin{array}{l}\text { Girish Bathla } \\
\text { girishmamc@gmail.com }\end{array}$} & 2 Univ & Iowa, 200 Hawkins Dr, Iowa City, IA 52242, USA \\
\hline \multirow{2}{*}{\multicolumn{2}{|c|}{$\begin{array}{l}\text { Michael Achilleos } \\
\text { Michael.achilleos@utsouthwestern.edu }\end{array}$}} & 3 Dep & f Radiology, University of Texas Southwestern \\
\hline & & $\mathrm{Me}$ & er, 5323 Harry Hines Blvd, Dallas, TX 75390, USA \\
\hline
\end{tabular}


However, it is now well established that this is a multi-system inflammatory process often presenting with acute cardiac events like myocarditis, an acute renal event, and multiorgan failure. Though uncommon, neurological manifestations in COVID-19 are increasingly being recognized. Although a wide range of neurological manifestations have been reported in the literature, ischemic stroke remains the most common subset of neurological manifestations in COVID, likely due to the associated hypercoagulable state. The second subset of neurological presentation involves a response to the cytokine storm and multi-system inflammation including acute demyelination, vasculitis, necrotizing encephalopathy, and posterior reversible encephalopathy syndrome. We report five cases with neuroimaging findings in COVID19 positive patients, presenting to the ER. In at least three of these, the primary presenting symptoms were neurological rather than respiratory.

\section{Clinical cases}

\section{Case 1: Ischemic stroke with large vessel occlusion}

A 41-year-old female presented with acute onset right-sided weakness and aphasia. Code stroke was activated with emergent head CT revealing a moderate sized left middle cerebral artery (MCA) territory infarct. CT angiogram showed occlusion of the M1 segment of left MCA with poor distal reconstitution. Large ischemic penumbra was noted on the CT perfusion maps with high mismatch ratio (Fig. 1a-d). Mechanical thrombectomy via a combination of aspiration and stent treatment combined with aspiration was performed with successful revascularization (Fig. 1e-f). The patient was incidentally tested for COVID and was found to be positive. Chest radiograph done on the same day showed minimal hazy bilateral infiltrates. It was concluded that the ischemic stroke was likely secondary to the dysregulation in the coagulation system and the increased pro-inflammatory state. No other underlying case for stroke could be identified. Over the course of the next week, however, there was significant worsening of respiratory symptoms and chest imaging findings with new pulmonary infiltrates. The patient was transferred to the Tactical COVID Unit (TCU) for further management and recovered.

\section{Case 2: Ischemic stroke with embolic infarcts}

A 78-year-old man with a history of prostate cancer, chronic hypertension, and recent positive diagnosis of COVID-19 presented with altered mental status and hypoxia. There was a strong clinical concern for stroke and venous thromboembolic event. Given the positive D-dimer and altered mental status, $\mathrm{CT}$ angiogram of the chest and MRI of the brain was recommended. Although the chest CT angiogram was negative for pulmonary thromboembolism, there were multi-focal consolidative and ground-glass pulmonary opacities, in a distribution consistent with COVID (Fig. 2a, b). Subsequent MRI revealed multiple punctate acute infarcts in multiple vascular territories suggesting underlying embolic source (Fig. 2c-e). Neurology was consulted for the abnormal MRI brain results with potential etiologies including hypercoagulable state in setting of active infection/inflammation versus underlying cardioembolic source. Over the next 2 weeks, his hospital course was further complicated by the development of shock liver, acute renal failure, and refractory cardiogenic shock, eventually resulting in patient's death.

\section{Case 3: Acute tumefactive demyelination}

A 33-year-old with morbid obesity and diabetes mellitus presented to the ER with hypoxemic respiratory failure secondary to COVID-19 status post-emergent intubation. The patient developed acute encephalopathy with extensor posturing. MRI revealed extensive tumefactive foci of demyelination with restricted diffusion and enhancement (Fig. 3a-f), most prominently involving the corpus callosum and the pericallosal white matter. The imaging findings were presumed to represent acute demyelinating injury from concurrent SARS-CoV-2 infection, given the monophasic appearance of the lesions in the setting of encephalopathy. CSF analysis showed no signs of viral infection or inflammation. The spectrum of clinicoradiological findings was consistent with primary damage from COVID-induced metabolic disruption. The patient completed a course of high-dose pulse steroids with IV methylprednisolone $(1 \mathrm{~g} \times 5$ days $)$ followed by a prolonged taper with minimal clinical improvement.

\section{Case 4: Cytotoxic lesion of the corpus callosum}

A 41- year-old female with a past medical history significant for diabetes, morbid obesity, and chronic kidney disease presented to the ER with respiratory failure secondary to SARSCoV-2 positive interstitial pneumonia. Following intubation, she was started on remdesivir. She had internuclear ophthalmoplegia and paralysiswith unequal pupils. Head CT revealed edema involving the posterior body and splenium of corpus callosum (Fig. 4a-c). Imaging findings were characteristic of transient cytotoxic lesion of the corpus callosum (CLOCCs). The scan also showed minimal parietal convexity subarachnoid hemorrhage (non-aneurysmal pattern) (Fig. 4d). Neurosurgical consult was obtained, and it was assumed that the SAH could be secondary to platelet dysfunction, seen in COVID patients. Edema involving the corpus callosum was assumed to be secondary to the COVID-related cytokine storm. There was marked clinical decline overnight with worsening acidosis and shock. She sustained cardiac arrest with pulseless electrical activity the next morning. 


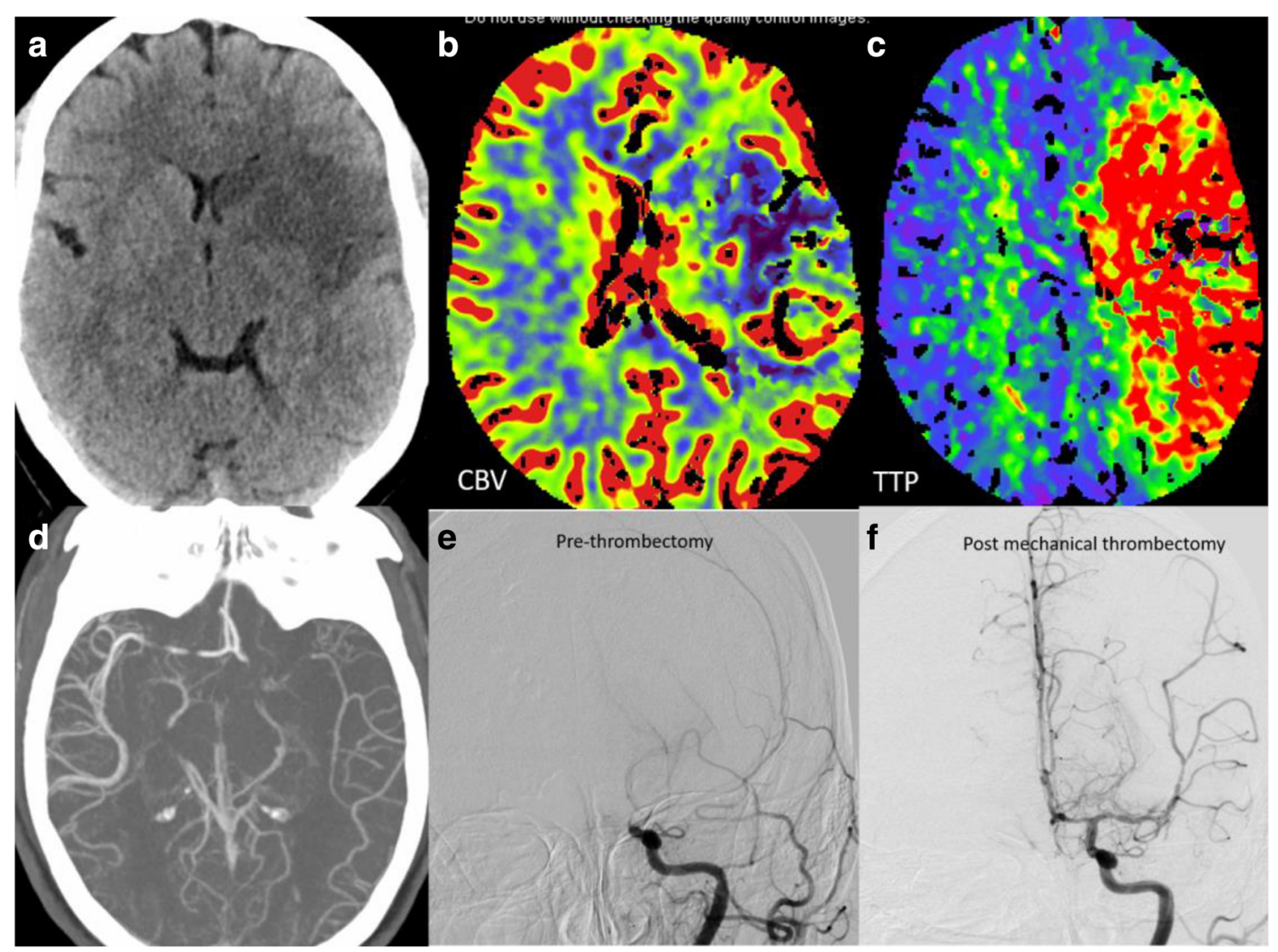

Fig. 1 Ischemic stroke with large vessel occlusion: Non-contrast head (a) shows moderate size acute infarct in the left MCA territory. Cerebral blood volume (CBV) map (b) shows corresponding acute infarct core with Time to Peak (TTP) map (c) showing large area of ischemic penumbra. CT and catheter angiogram images depicting the left MCA thrombotic occlusion (d, e) with significant revascularization post mechanical thrombectomy (f)
Fig. 2 Ischemic stroke with embolic infarcts: Chest CT images $(\mathbf{a}, \mathbf{b})$ reveal multi-focal consolidative and ground-glass pulmonary opacities, along with areas of crazy paving suggesting acute lung injury. Multiple diffusion-weighted images $(\mathbf{c}-\mathbf{e})$ show acute punctate embolic infarcts (arrows)

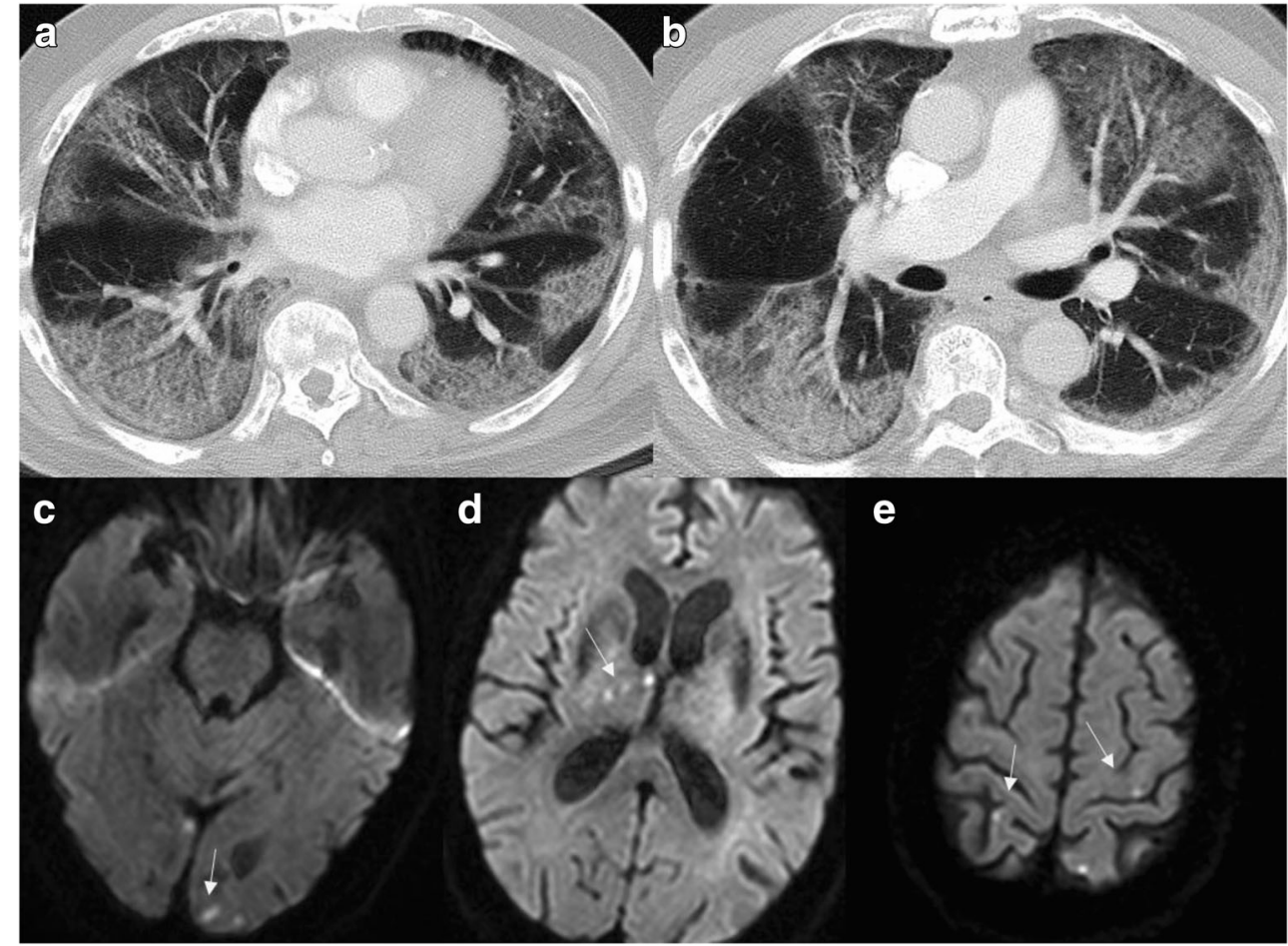


Fig. 3 Acute tumefactive demyelination: Sagittal T1 (a), sagittal T2-FLAIR (b), axial T2FLAIR (c), diffusion-weighted image (d), axial (e), and coronal (f) post-contrast images reveal extensive foci of demyelination in the supratentorial brain with marked involvement of the corpus callosum and pericallosal white matter. Many of these lesions show restricted diffusion and patchy enhancement (arrows)

Fig. 4 Cytotoxic lesion of the corpus callosum: Multiple CT head images reveal edema with hypodensity involving the posterior body and splenium of corpus callosum (white arrows) Minimal subarachnoid hemorrhage seen along right parietal convexity (black arrow)
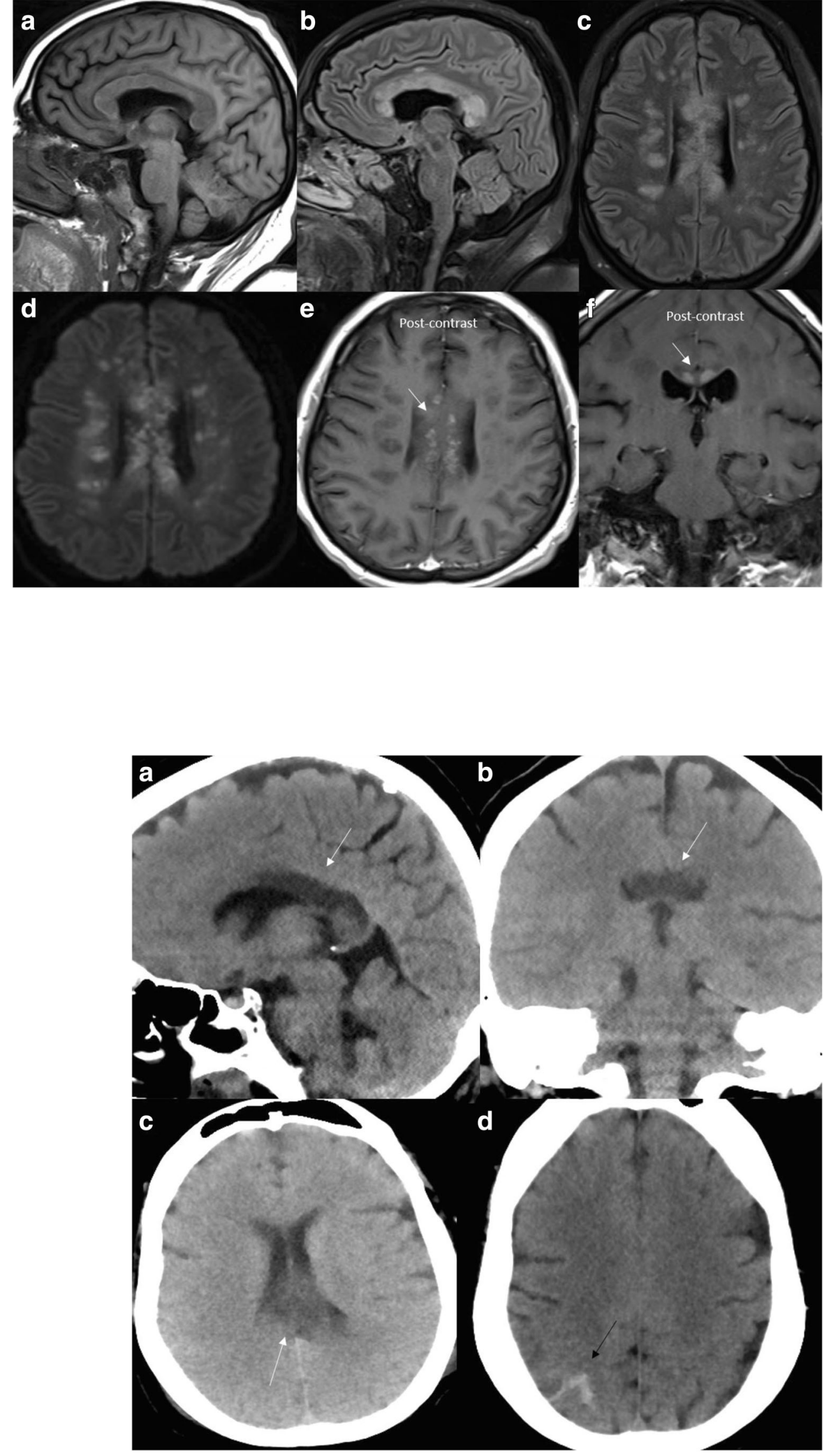


\section{Case 5: Posterior reversible encephalopathy syndrome}

A 27-year-old female presented to the ER with dizziness and vertigo for the last week. Emergent chest CT (to rule out pulmonary thromboembolism) and head CT were unremarkable. She was transferred to the dedicated COVID ICU after being found SARS-CoV-2 RT-PCR positive. In the meantime, patient had significant clinical deteriorating, became encephalopathic, and developed rapidly worsening acute liver failure. ID was consulted for recommendations in the setting of possible liver transplant and COVID-19 positive status. Subsequent MRI revealed subcortical white matter signal changes characteristic of posterior reversible encephalopathy syndrome (Fig. 5a-d). The underlying etiology for this was assumed to be either the cytokine storm seen in COVID or secondary to metabolic derangement as seen in acute liver failure. Patient expired after 2 weeks due to cardiopulmonary arrest in the setting of fulminant liver failure and shock.

\section{Discussion}

The H1N1 influenza flu of 1918, also known as Spanish flu, was one of the worst pandemics known to mankind, wherein more than 500 million people were infected [1]. More than a century later, the world witnessed another major pandemic: novel Severe Acute Respiratory Syndrome-coronavirus 2 pandemic, simply known as COVID-19.

The SARS-CoV-2 like other coronavirus primarily affects the pulmonary system, with respiratory failure being the cause for death in the majority of patients. The most established pattern on chest imaging includes multi-focal areas of consolidation in a peripheral distribution pattern [2]. As our knowledge about the disease is expanding, it has become clear that other organ systems are also commonly involved, most prominently, involving acute cardiac and renal complications. Neurological complications are also relatively common ranging from 30 to $45 \%$ depending upon the severity of the disease, as reported in one of the first studies on the subject by Mao et al. [3] These symptoms could be fairly specific like anosmia, ischemic, or hemorrhagic stroke or more nonspecific like mental status changes, headache, and seizures.. A wide range of neuroimaging manifestations have now been reported, mainly in the form of case reports and case series. Recently, some original research articles have also been published. Mahammedi et al. [4] published a large study with 725 consecutive hospitalized COVID-19 patients, with 108 patients having neurological complications. To our knowledge, this was the first study to systematically characterize neurological symptoms and neuroimaging findings in COVID-19 patients. Table 1 provides a summary of the most common
Fig. 5 Posterior reversible encephalopathy syndrome (PRES): axial T2-FLAIR images $(\mathbf{a}, \mathbf{b})$ and axial T2-spine echo image (c) reveals near-symmetric areas of subcortical signal changes with edema and sulcal effacement in the occipital lobes. Apparent diffusion coefficient (ADC) maps (d) show increased signal representing facilitated diffusion as seen in vasogenic edema

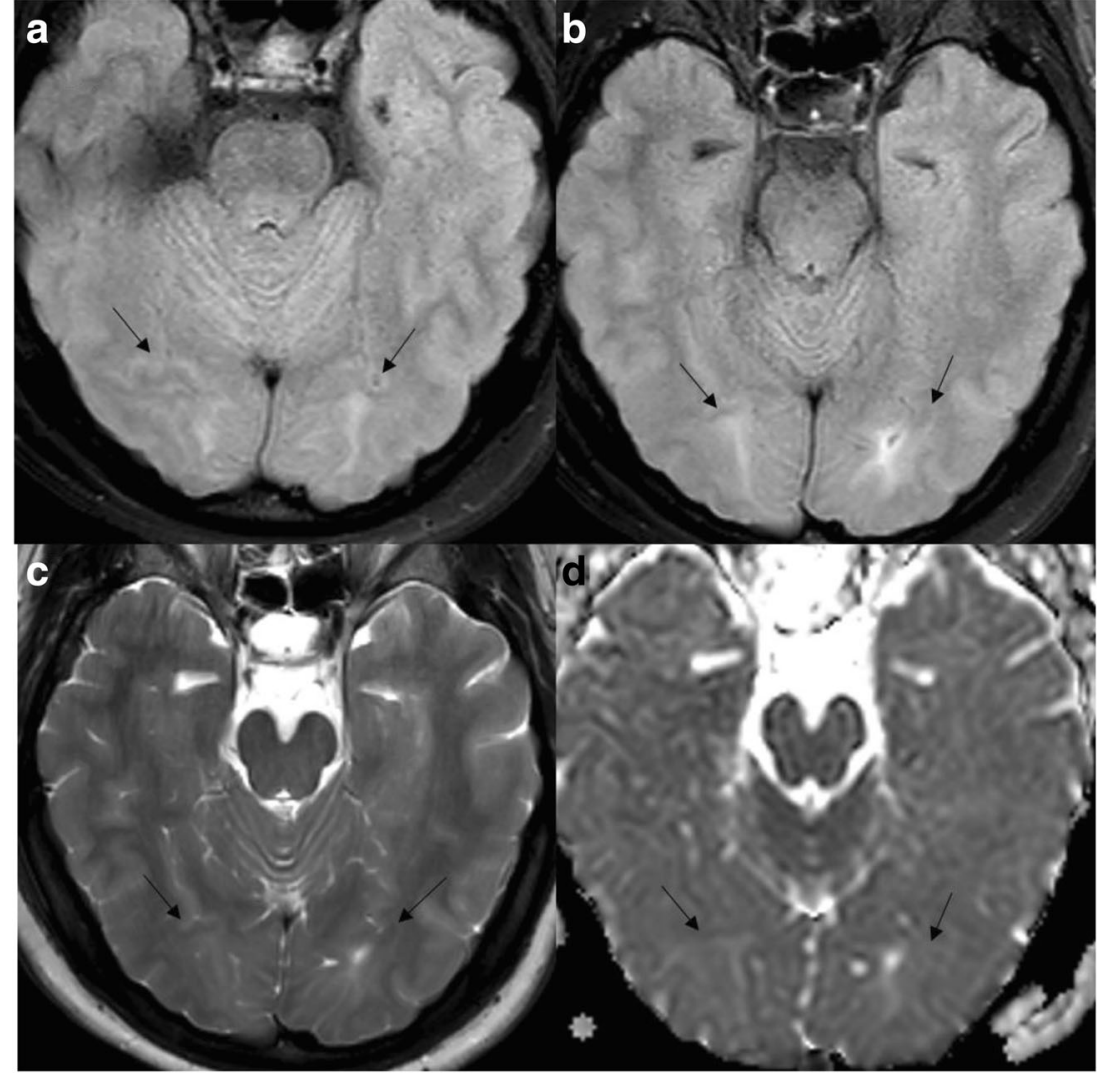


Table 1 Spectrum of neuroimaging manifestations of COVID-19

\begin{tabular}{|c|c|c|c|}
\hline Complication & Number* & Imaging findings & Authors \\
\hline \multicolumn{4}{|l|}{ Hypercoagulable state } \\
\hline Ischemic stroke & 34 & Large vessel territorial, small-vessel and embolic infarcts & Mahammedi et al. [4] \\
\hline $\begin{array}{l}\text { Cerebral venous sinus } \\
\text { thrombosis }\end{array}$ & 3 & Occlusion of major dural venous sinuses with associated parenchymal changes & Cavalcanti et al. [5] \\
\hline \multicolumn{4}{|c|}{ Neurotropism and other pro-inflammatory state complications } \\
\hline $\begin{array}{l}\text { Diffuse leukoencephalopathy } \\
\text { with or without } \\
\text { microhemorrhages }\end{array}$ & 11 & $\begin{array}{l}\text { Symmetric confluent white matter T2 hyperintensity and restricted diffusion } \\
\text { with relative sparing of juxtacortical and infratentorial white matter, with or } \\
\text { without microhemorrhages }\end{array}$ & Radmanesh et al. [6] \\
\hline Leptomeningitis & 8 & Leptomeningeal enhancement or FLAIR sulcal hyeritensity & Helms et al. [7] \\
\hline Encephalitis/Encephalopathy & 22 & $\begin{array}{l}\text { T2 FLAIR hyperintensity within the bilateral medial temporal lobes, basal } \\
\text { ganglia and thalami with or without hemorrhage }\end{array}$ & Scullen et al. [8] \\
\hline Acute demyelination & 4 & $\begin{array}{l}\text { Non-confluent multifocal white matter hyperintense lesions on FLAIR and } \\
\text { diffusion, with variable enhancement }\end{array}$ & Kremer et al. [9] \\
\hline $\begin{array}{l}\text { Posterior reversible } \\
\text { encephalopathy syndrome } \\
\text { (PRES) }\end{array}$ & 2 & Symmetric vasogenic edema within the occipital and parietal regions & Franceschi et al. [10] \\
\hline Injury to the olfactory bulbs & 5 & Microbleeding or abnormal enhancement within the olfactory bulbs on MR & Aragão [11] \\
\hline Gullian-Barre syndrome & 1 & $\begin{array}{l}\text { Thickening and contrast enhancement on the conus medullaris and the cauda } \\
\text { equine nerve roots }\end{array}$ & Zao [12] \\
\hline Intracranial hemorrhage & 6 & Parenchymal or subarachnoid hemorrhage & Mahammedi et al. [4] \\
\hline Vasculitis & 1 & Vasculitis pattern infarcts and/or vessel wall enhancement & Hanafi et al. [13] \\
\hline Transient cytotoxic edema & 4 & $\begin{array}{l}\text { T2-FLAIR hyperintense lesions involving both middle cerebellar peduncles or } \\
\text { splenium of corpus callosum }\end{array}$ & Kremer et al. [9] \\
\hline $\begin{array}{l}\text { Exacerbation of multiple } \\
\text { sclerosis }\end{array}$ & 2 & Clinical and imaging progression of demyelinating plaques & Mahammedi et al. [4] \\
\hline
\end{tabular}

$*_{n}=$ number of cases reported in the series

neuroimaging manifestations reported to date. There are two broad sets of neurological complications in COVID-19: (1) vascular complications with stroke secondary to arterial or venous thrombosis, related to the known hypercoagulable state seen in COVID [4, 5, 14], and (2) much broader gamut including diffuse leukoencephalopathy, acute demyelination, posterior reversible encephalopathy syndrome (PRES), necrotizing encephalopathy, and focal cytotoxic edema, primarily seen as a consequence of systemic inflammation and cytokine storm seen with COVID-19 [6-13].

\section{Hypercoagulable state}

There is growing evidence and consensus that COVID-19 infection is associated with a pro-thrombotic state, leading to an increased incidence of arterial and venous thrombosis and microvascular coagulopathy [15]. The cumulative incidence of venous thromboembolism (25-49\%), acute ischemic stroke (0.9-5\%), and acute myocardial injury (up to 20\%) further underscore the coagulopathic effects of COVID-19 [3, 15, 16]. The increased thrombotic tendency is likely multifactorial. Immune hyperactivation and cytokine storm in severe COVID-19 are now well recognized and likely result from the cytopathic effects of the virus. Cerebral arterial or venous thrombosis is at present the most commonly reported neurological complication of COVID [4]. Acute ischemic stroke in COVID-19 may be seen at presentation in the ER or during the course of the hospitalization, generally seen in patients with severe disease who often require intensive care. Stroke may be the presenting symptom or reason for admission in up to $44-46 \%$ of patients presenting with neurological complications [6]. Dural venous sinus thrombosis is also increasingly being observed, with the largest series of three patients reported by Cavalcanti et al. [5]

Arterial thrombi can involve medium and large-sized arteries across the body. In a study, by Dane et al., $9(11 \%)$ out of a cohort of 82 patients had thromboembolic findings in the abdomen, pelvis, and lower extremities. [17] The etiology of stroke is generally cryptogenic, with involvement of both the anterior and posterior circulation vessels. Large-vessel anterior circulation involvement is the most common observed pattern of ischemic stroke in COVID patients. [6]

These patients are often put on empirical full dose of anticoagulants, given the significant hypercoagulability in COVID-19. Interestingly, the incidence of ischemic strokes in COVID-19 may be decreasing after the initial peak, possibly due to routine anticoagulation protocols in critically ill 
patients with higher d-dimer levels [4, 17]. However, this may be temporary, given the current trend and increasing number of positive cases in the USA.

\section{Neurotropism and other pro-inflammatory state complications}

Neurotropism of SARS-CoV-2 and the sensitivity and specificity of its detection in the CSF have not been well established. The virus has been detected through RT-PCR in CSF samples and also in brain tissue on autopsy, suggesting high possibility that the virus can breach the blood brain barrier. COVID has known affinity for ACE2 receptors, also expressed by glial cells and neurons, making them potential targets of the virus. $[18,19]$ Very recently, Aragão et al. reported a series of five patients with hemorrhage or postcontrast enhancement within the olfactory bulbs on MRI, three of which presented with anosmia. This was proposed to be secondary to trans-synaptic transfer of the virus through the cribriform plate. [11]

The CNS manifestations could be due to the direct effect of the virus on the brain or secondary to immune-mediated changes as seen with other viral illnesses like H1N1. COVID-19 is known to cause a severe cytokine storm with resultant pro-inflammatory state mediated predominantly by IL1, IL-6, and TNF $\alpha$. $[18,19]$ Acute monophasic demyelination, like acute disseminated encephalomyelitis; tumefactive demyelination; and acute exacerbation of multiple sclerosis have now been reported with COVID-19. The exact mechanism is again unclear. $[3,4,7]$ The wide spectrum of neurological complications includes conditions like Guillain-Barré syndrome, cranial nerve enhancement, similar to other viral neuropathies $[12,20]$. Vasculitis or leptomeningits could again be secondary to the cytokine storm or due to direct infection and inflammation of these structures [7, 13]. In a relatively large series, Radmanesh et al. reported COVIDassociated leukoencephalopthy (with our without microhemorrhage) in 11 patients and proposed delayed posthypoxic insult as the underlying mechanism, similar to what is seen in drug overdose and cardiac arrest [6]. This second set of neurological complications in COVID-19 is less well defined with a very wide spectrum and subject to multiple other overlapping causative factors. $[4,10]$

\section{Conclusion}

The spectrum of neurological manifestations in COVID-19 is very wide at present. Understanding the neurological manifestations in COVID-19 is still at a very nascent phase. The most common neurological presentation reported has been ischemic stroke, secondary to arterial or venous thrombosis, because of the hypercoagulable state associated with COVID-19. However, the neurological manifestation can be quiet variable, as depicted in this paper.

\section{Compliance with ethical standards}

Conflict of interest The authors declare that they have no conflict of interest.

\section{References}

1. Jester B, Uyeki T, Jernigan D (2018) Readiness for responding to a severe pandemic 100 years after 1918. Am J Epidemiol 187(2): 2596-2602

2. Salehi S, Abedi A, Balakrishnan S, Gholamrezanezhad A (2020) Coronavirus disease 2019 (COVID-19): a systematic review of imaging findings in 919 patients. Am J Roentgenol 215(1):87-93

3. Mao L, Jin H, Wang M et al (2020) Neurologic manifestations of hospitalized patients with coronavirus disease 2019 in Wuhan China. JAMA Neurol 77(6):1-9

4. Mahammedi A, Saba L, Vagal A, Leali M., Rossi A., Gaskill M., Sengupta S., Zhang B., Carriero A., Bachir S., Crivelli P., Paschè A., Premi E., Padovani A., Gasparotti R. (2020) Imaging in neurological disease of hospitalized COVID-19 patients: an Italian multicenter retrospective observational study. Radiology. 201933

5. Cavalcanti DD, Raz E, Shapiro M, et al. (2020) Cerebral venous thrombosis associated with COVID-19. AJNR Am J Neuroradiol

6. Radmanesh A, Derman A, Lui YW, et al. (2020) COVID-19 -associated diffuse leukoencephalopathy and microhemorrhages. Radiology 21

7. Helms J, Kremer S, Merdji H et al. (2020) Neurologic features in severe SARS-CoV-2 infection. N Engl J Med 15

8. Scullen T, Keen J, Mathkour M, Dumont AS, Kahn L (2020) COVID-19 associated encephalopathies and cerebrovascular disease: the New Orleans experience [published online ahead of print, 2020 may 28]. World Neurosurg 20(3):S1878-S8750

9. Kremer S , Lersy F, Sèze J. (2020) Brain MRI Findings in severe COVID-19: a retrospective observational study. Radiology 16

10. Franceschi A, Ahmed O, Giliberto L, Castillo M. (2020) Hemorrhagic posterior reversible encephalopathy syndrome as a manifestation of COVID-19 infection. AJNR Am J Neuroradiol 21

11. Aragão M, Leal M, Filho O, et al. (2020) Anosmia in COVID-19 associated with injury to the olfactory bulbs evident on MRI. AJNR Am J Neuroradiol 25

12. Zhao H, Shen D, Zhou H, Liu J, Chen S (2020) Guillain-Barrè syndrome associated with SARS-CoV-2 infecion: causality or coincidence? Lancet Neurol 19(5):383-384

13. Hanafi R, Roger P, Perin B, et al. (2020) COVID-19 neurologic complication with CNS vasculitis-like pattern. AJNR Am J Neuroradiol 18

14. Lodigiani C, Iapichino G, Carenzo L, Cecconi M, Ferrazzi P, Sebastian T, Kucher N, Studt JD, Sacco C, Alexia B, Sandri MT, Barco S (2020) Venous and arterial thromboembolic complications in COVID-19 patients admitted to an academic hospital in Milan, Italy. Thromb Res 191:9-14

15. McFadyen JD, Stevens H, Peter K. (2020) The emerging threat of (micro)thrombosis in COVID-19 and its therapeutic implications. Circ Res 26

16. Klok FA, Kruip M, Meer N et al (2020) Incidence of thrombotic complications in critically ill ICU patients with COVID-19. Thromb Res 191:145-147 
17. Dane B, Smereka P, Wain R, et al. (2020) Hypercoagulability in COVID-19: identification of arterial and venous thromboembolism in the abdomen, pelvis, and lower extremities. AJR Am J Roentgenol 29

18. Zhong J, Tang J, Ye C, Dong L (2020) The immunology of COVID-19: is immune modulation an option for treatment? Lancet Rheumatol 2(7):e428-e436

19. Netland, J, Meyerholz, D, Moore,S, et al. Severe acute respiratory syndrome coronavirus infection causes neuronal death in the absence of encephalitis in mice transgenic for human ACE2. J Virol 2008; 82 (15):7264-7265
20. Román GC, Spencer PS, Reis J et al (2020) The neurology of COVID-19 revisited: a proposal from the Environmental Neurology Specialty Group of the World Federation of Neurology to implement international neurological registries. $J$ Neurol Sci 414:116884

Publisher's note Springer Nature remains neutral with regard to jurisdictional claims in published maps and institutional affiliations. 